\title{
Uma proposta experimental em mecânica quântica: uns fótons, uma fenda, uma foto
}

An experimental proposal in quantum mechanics: some photons, a slit, a photo

\author{
J. J. O. Silva ${ }^{1}$, E.S. Rodrigues ${ }^{2}$, D. M. S. Ribeiro ${ }^{3}$, M. S. Silva ${ }^{4}$ \\ Instituto Federal de Educação Ciência e Tecnologia do Sertão Pernambucano, CEP 56.000-000, Salgueiro- \\ Pernambuco, Brasil \\ $\left\{{ }^{1}\right.$ junior.oliveira, ${ }^{2}$ eriverton.rodrigues, ${ }^{3}$ daiane.ribeiro, ${ }^{4}$ marcelo.silva $\} @$ ifsertao-pe.edu.br \\ (Recebido em 23 de março de 2016; aceito em 28 de setembro de 2016)
}

Este trabalho propõe uma metodologia para atividades práticas, envolvendo conhecimentos de programação e conceitos de física quântica, para obter dados relativos ao padrão de difração de Fraunhofer e, assim, estudar um dos princípios que fundamentam a mecânica quântica: o Princípio da Incerteza de Heisenberg. Com a utilização didática de recursos tecnológicos contemporâneos para o ensino de tópicos de física moderna, espera-se promover um ambiente promissor para o ensino de temas que se relacionem com a ciência, tecnologia e informação. Foi proposto um algoritmo para aquisição de dados referentes às distancias entre os mínimos de intensidade no padrão de difração, embora o algoritmo não tenha sido aplicado em sala de aula esse trabalho propõe a comparação dos dados obtidos pelo algoritmo e por medidas diretas feitas com uma régua. A utilização dos recursos sugeridos neste trabalho deve envolveras disciplinas de Física e linguagem de programação, podendo ser aplicados, por exemplo, em cursos de nível médio integrado em informática, ou em disciplinas como metodologia para o ensino de Física em cursos de licenciatura em Física. A automação dos dados permitiu estudar o princípio da incerteza e obter o valor da constante de Planck de forma alternativa à medida direta da distância entre os mínimos de difração.

Palavras-chave: princípio da incerteza, difração, algoritmo computacional

This paper proposes a methodology for practical activities involving programming knowledge and concepts of quantum physics, to gather data related to Fraunhofer diffraction standard and thus to study one of the principles that underlie quantum mechanics: the Heisenberg uncertainty principle. Through the didactic use of contemporary technological resources for teaching modern physics topics, it is expected to promote a promising environment for teaching subjects that are related to science, technology and information. An algorithm for data acquisition concerning the distances between the intensity minimum in the diffraction pattern was proposed, although the algorithm has not been implemented in the classroom this work proposes to compare the data obtained by the algorithm and by direct measurements with a ruler. We objectify in this paper the use of resources involving disciplines of physics and programming language, can be applied, for example, mid-level integrated into computer courses, or in disciplines such methodology for physics teaching in undergraduate courses in physics. The automation of the data allowed to study the uncertainty principle and get the value of Planck's constant alternatively the direct measurement of the distance between the minimums of diffraction.

Keywords: uncertainty principle, diffraction, computational algorithm

\section{INTRODUÇÃO}

A Mecânica Quântica é um dos principais pilares das tecnologias contemporâneas. Seus conceitos são aplicados na construção e no desenvolvimento dos mais diversos dispositivos tecnológicos, que vão de transistores a fotossensores e de LEDs a memórias magnéticas [1]. Apesar da computação quântica de dados e da transmissão quântica de informações serem temas efervescentes no panorama científico atual, é notório que o estudo de tais temas ainda é restrito a minorias, praticamente apenas os estudantes que resolvem seguir carreiras acadêmicas voltadas a algumas áreas científicas entram em contato com esses temas e conceitos, mesmo assim, em geral, no fim dos seus cursos de graduação.

Este artigo propõe uma abordagem didática transversal para ser aplicada ao ensino médio ou aos primeiros semestres dos cursos universitários que envolva temas da mecânica quântica e noções de programação para processamento de imagens, considerando a relevância da participação dos estudantes nas atividades didáticas de caráter prático [2]. 
O conceito de quantização, relacionado à constante de Planck, representa uma mudança na compreensão humana sobre o comportamento de sistemas microscópicos, tornando-se um marco na evolução das ciências. Ao fim do século XIX, a física estava bem-estabelecida com base no eletromagnetismo clássico, mecânica clássica e na termodinâmica. Apoiada nessas teorias, as tentativas de explicar o comportamento da interação da radiação e matéria não foram bemsucedidas [3].

A ideia da emissão ou absorção da energia quantizada surgiu no desenvolvimento de cálculos como condição de compatibilidade entre valores da entropia e de um sistema microscópico [4]. As teorias clássicas preveem um comportamento de partículas oscilantes dentro de uma pequena caixa de modo que, para uma dada temperatura, qualquer radiação possa ser emitida com qualquer comprimento de onda. No entanto a condição proposta pela teoria quântica é mais radical na medida em que as partículas oscilando só poderiam emitir radiação em pacotes discretos de energia $(E)$ [4]. Não obstante, surgem ideias também revolucionárias segundo as quais, as partículas podem apresentar comportamento de onda; e as ondas, características de partículas, ou seja, uma "onda eletromagnética" se propaga como onda, mas interage com a matéria como partícula [5].

Este trabalho se destina a construir uma proposta de atividade prática voltada ao estudo de conceitos relacionados à física moderna no ensino médio ou nos primeiros semestres de cursos superiores $[14,15]$. Neste caso, temos uma interdisciplinaridade entre física e informática, através do estudo da difração de um laser pela fenda de um instrumento simples como o paquímetro, permitindo controle da abertura da fenda como variável, o que possibilita a observação da dependência do padrão de difração em função da espessura da fenda diferentemente da utilização do $C D$ como rede de difração $[8,13]$. Assim, a implementação de um algoritmo para a obtenção de dados se configura em um desafio que pode ser proposto ao estudante e pode servir de cenário para o estudo desses conceitos. Na Seção 2 apresenta-se a metodologia para uma possível implementação do algoritmo. Entre os princípios fundamentais da mecânica quântica, destaca-se o princípio da incerteza de Heisenberg [6], segundo o qual existe uma limitação na precisão, imposta pelo próprio processo de medida. Por exemplo: determinar simultaneamente a posição $(x)$ e a componente $x$ do momento $\left(p_{x}\right)$ de uma partícula tem sua incerteza mínima determinada por:

$$
\Delta x . \Delta p_{x} \geq h
$$

Nessa relação, $\Delta x$ representa a incerteza na medida da posição e $\Delta p_{x}$ é a incerteza na componente $x$ do momento da partícula e h é a constante de Planck $\left(h=6.6262 \times 10^{-34} \mathrm{~J}\right.$.s $)$ dividida por $2 \pi[7]$.

\section{Difração de fraunhofer por fenda simples.}

A difração de Fraunhofer é o efeito de difração de uma fonte luminosa distante suficientemente para garantir que as frentes de ondas sejam planas e incidam perpendicularmente à fenda. Para que ocorra a difração, o comprimento de onda da radiação incidente deve ter uma ordem de grandeza próxima a do comprimento de onda, é possível considerar a fenda iluminada como sendo uma distribuição de irradiadores elementares emitindo luz em todas as direções. Portanto, a luz sofrerá interferência tanto construtiva quanto destrutiva e irá formar no anteparo franjas de interferência, conforme ilustra a Figura 1. 


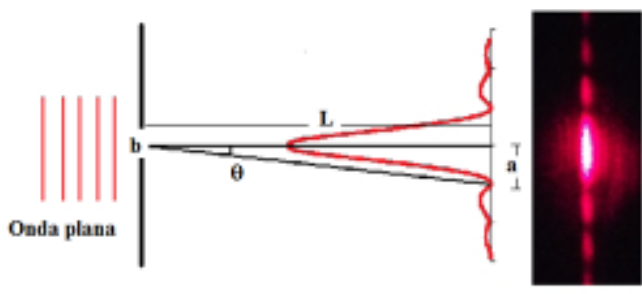

(a)

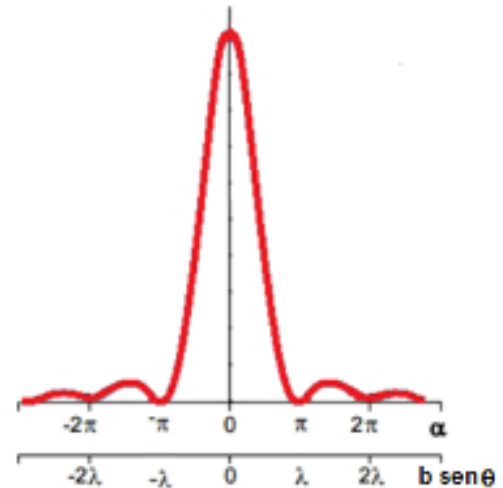

(b)

Figura 1: Ilustração do padrão de difração associado aos pontos de máximo da intensidade luminosa. (a) parâmetros relacionados à montagem do aparato, (b) gráfico de intensidade luminosa em função do ângulo. (Fonte: Do autor 2015)

Na Figura 1-(a) "L" representa a distância entre a fenda e o anteparo, "b", a espessura da fenda e " $\theta$ " é o ângulo entre o máximo central e o ponto $(P)$ do anteparo onde se pretende medir a intensidade. A intensidade da luz que atinge o anteparo é dada pela relação:

$$
\mathrm{I}=\mathrm{I}_{0} \frac{\operatorname{Sen}^{2}(\alpha)}{\alpha^{2}}
$$

Nessa expressão, $I_{0}$ é a intensidade luminosa que atinge a fenda e $\alpha$ é um parâmetro angular depende do ângulo $(\theta)$, e da espessura da fenda (b), de acordo com a Equação (3):

$$
\alpha=\frac{k \cdot b \cdot \operatorname{Sen}(\theta)}{2}
$$

No ponto onde a intensidade é máxima, ou seja, $\mathrm{I}=\mathrm{I}_{0}$, deve-se ter $\alpha=0$, conforme ilustra a Figura 1- (b). Ao analisar a Equação (2), verifica-se que o ponto onde a intensidade luminosa é um mínimo e deve obedecer a chamada condição de mínimo, em que $\operatorname{sen}(\alpha)=0$, ou seja, $\alpha=\mathrm{m}$ $\pi$, com $\mathrm{m}$ sendo um número inteiro, positivo e diferente de zero. Sabendo que $k=\frac{2 \pi}{\lambda}$, é possível escrever:

$$
\begin{aligned}
& \mathrm{m} \pi=\frac{k \cdot b \cdot \operatorname{Sen}(\theta)}{2} \\
& m 2 \pi=\mathrm{k} \cdot b \cdot \operatorname{Sen}(\theta) \\
& \mathrm{m}=\frac{b}{\lambda} \cdot \operatorname{Sen}(\theta)
\end{aligned}
$$

Quando a onda plana é difratada pela fenda de largura $\Delta y=b$, a onda é dispersa em várias direções. Para ser desviado de forma a produzir no anteparo uma distribuição angular com abertura $\theta$, o momento do elétron deve adquirir uma componente vertical dada por: $\Delta p_{y}=$ $\operatorname{psen}(\theta)$. A relação de Broglie diz que $p=\frac{h}{\lambda}$, portanto:

$$
\Delta \mathrm{p}_{y}=\frac{h}{\lambda} \operatorname{sen}(\theta)
$$

Substituindo $\Delta y=b$ e a Equação 5 na Equação 4, obtemos:

$$
\Delta \mathrm{y} . \Delta \mathrm{p}_{y}=\mathrm{mh}
$$

Como $m$ é um número inteiro, o produto $\Delta \mathrm{y} . \Delta \mathrm{p}_{y}$ sempre será em módulo maior que $h$, assim confirmando o princípio da incerteza [12]. Abre-se uma oportunidade de discutir o caráter dual 
da luz, pois, ao considerar o fóton apenas uma partícula, a distribuição da intensidade luminosa deveria ser gaussiana em torno do centro, sem apresentar máximos e mínimos. Contudo o fenômeno de difração é típico de ondas, e as propriedades desse fenômeno permitem verificar a Equação 4, essa relação, obtida de forma muito simplificada, está próxima ao resultado mais rigoroso expresso pela Equação 1. Trata-se de uma maneira simples de estudar o Princípio da Incerteza sem a necessidade de instrumentos laboratoriais e/ou formalismos matemáticos muito rebuscados [12].

\section{MATERIAL E MÉTODOS}

$\mathrm{O}$ aparato foi montado fazendo incidir a luz proveniente de um laser de diodo com comprimento de onda $\lambda=670 \mathrm{~nm}$, sob a fenda de um paquímetro, conforme é apresentado na Figura 2. Ao variar a espessura da fenda é possível observar a alteração no padrão de difração que se forma em um anteparo distante $3,4 \mathrm{~m}$ do paquímetro no lado oposto ao lado da incidência do laser.

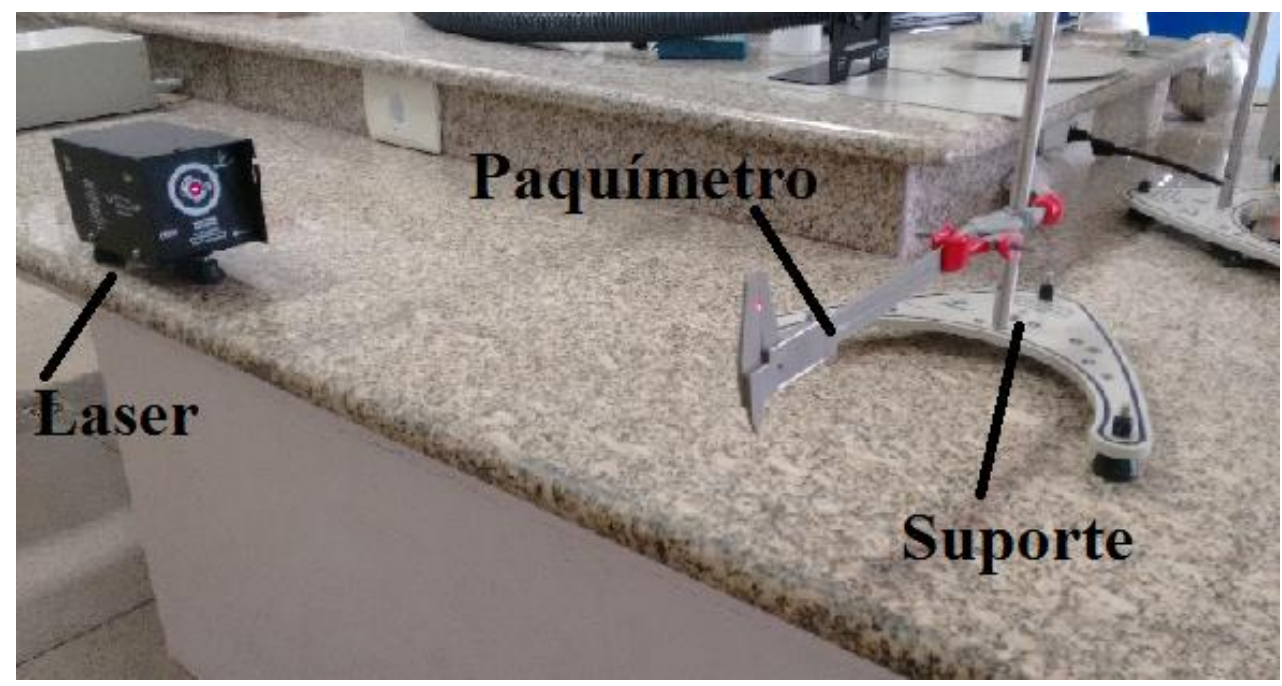

Figura 2: Montagem do aparato experimental. (Fonte: Do autor, 2016)

O gráfico de intensidade foi obtido fotografando o padrão de difração com uma máquina fotográfica Olympus de 12 megapixels. Utilizando o software Matlab R2014a [9], foi implementado um algoritmo para criar o gráfico da intensidade da luz em função da posição, proveniente de cada imagem e, posteriormente, calcular a distância entre o primeiro mínimo local esquerdo e o primeiro mínimo local direito.

O Matlab (MATrix LABoratory) é um software especializado em cálculos com matrizes. Esse software possui sua própria linguagem de programação, a linguagem Matlab, que fornece uma variedade de funções em várias especialidades. Em alguns casos essas funções resolvem problemas complexos como equações diferenciais, inverter uma matriz, binarizar uma imagem, entre outros [11]. O Matlab fornece um conjunto de ferramentas para a manipulação de imagens, fato preponderante na escolha do software neste trabalho.

O objetivo do algoritmo proposto neste trabalho foi automatizar o cálculo da distância entre o primeiro mínimo local esquerdo e o primeiro mínimo local direito. O conjunto de passos, usados pelo algoritmo, é descrito a seguir.

\section{ALGORITMO}

O algoritmo, escrito na linguagem Matlab encontra-se no repositório GitHub [16]. A Figura 3 ilustra o conjunto de passos seguido pelo algoritmo partindo da conversão da imagem em RGB para tons de cinza, até a criação do gráfico de intensidade luminosa do padrão de difração 
projetado no anteparo. Note-se que a imagem original na escala RGB, chamada de $I M_{0}$, é, inicialmente, convertida para a escala de tons de cinza ( 0 a 255), gerando a matriz $I M_{G S}$ e, em seguida, a imagem é binarizada. Após esse processo, a matriz binária $I M_{B I N}$ é usada para obter o gráfico de intensidade.

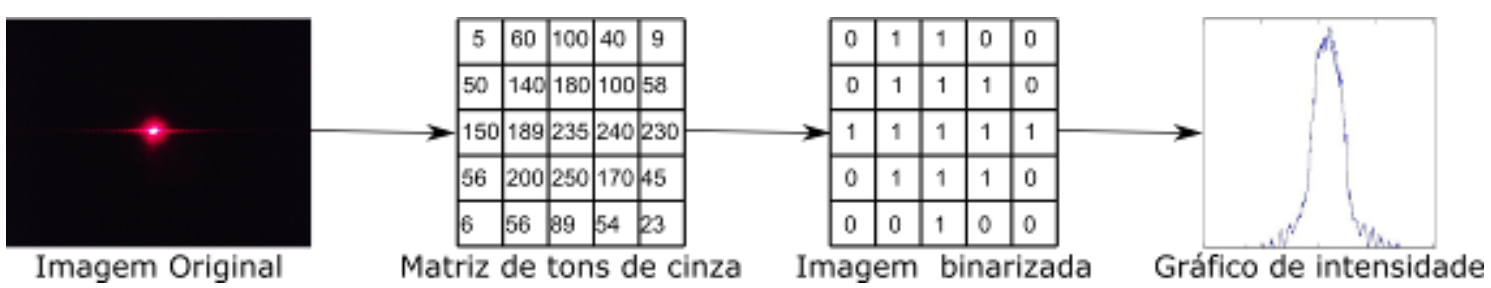

Figura 3: Processo de criação do gráfico de intensidade. (Fonte: Do autor 2015)

\section{Binarização}

O processo de binarização considera a matriz $I M_{G S}$. Se o valor em $I M_{G S}(i, j)(i$ e $j$ correspondem a linha $i$ e coluna $j$ de uma matriz, respectivamente) for maior que um limiar $\alpha I M_{B I N}(\mathrm{i}, j)$ recebe o valor 1, caso contrário, recebe o valor 0 (zero), como mostra a Equação (7). O Matlab fornece uma função computacional chamada $i m 2 b w(i m g, \alpha)$, que recebe uma imagem (img) e um limiar $\alpha$ (valor compreendido entre 0 (zero) e 1) e fornece como saída a imagem binária correspondente. Está função foi usada neste trabalho para obter a imagem binária.

Otsu [10] propôs um algoritmo para encontrar o valor de $\alpha$ dada uma imagem. Nos experimentos realizados neste trabalho, o algoritmo de Otsu não se adequou muito bem. Como pode-se perceber nas figuras (Figura 4-(a), Figura 4-(b), e Figura 4-(c)), a medida que o valor do limiar diminui, a separação entre pontos com maior e menor intensidade luminosa fica comprometida. Pelo contrário, ao aumentar muito esse valor, perde-se informações importantes. A partir de um conjunto de imagens de amostra, chegou-se à conclusão que o limiar 0,22 apresentou maior separação entre os pontos com maior e menor intensidade de luz.

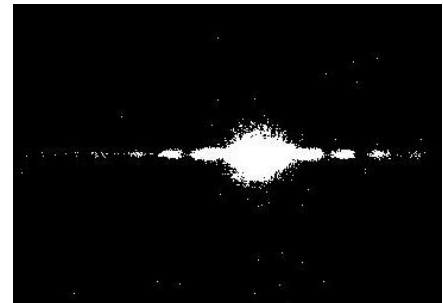

(a)

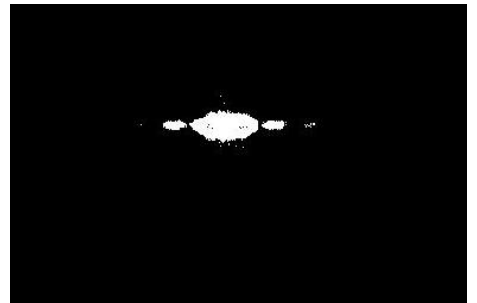

(b)

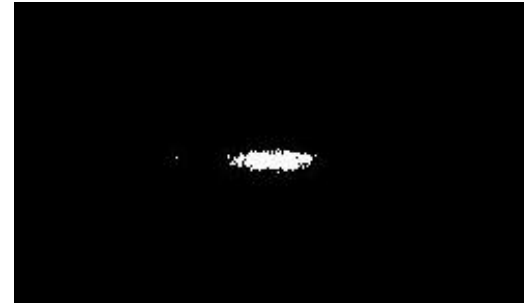

(c)

Figura 4: Binarização da imagem do padrão luminoso. Em (a) usou-se $\alpha=0,15$, em (b) $\alpha=0,22$ (padrão adotado neste trabalho) e em (c) usou-se o valor encontrado pelo algoritmo de Otsu. Note-se que o padrão apresentado em (b) obtém maior separação entre os mínimos. (Fonte: do autor 2016)

$$
\operatorname{IM}_{\mathrm{BIN}}(\mathrm{i}, \mathrm{j})=\left\{\begin{array}{lr}
1 & \text { se } \mathrm{IM}_{\mathrm{GS}}(\mathrm{i}, \mathrm{j})>\alpha \\
0 & \text { caso contrário }
\end{array}\right\}
$$

Gráfico

$\mathrm{O}$ algoritmo percorre cada coluna $j$ da matriz $\operatorname{IM}_{B I N}$ somando os valores contidos em cada linha $i$ correspondente a $j$. O valor da soma é armazenado em um vetor $G$ que representa o gráfico da intensidade da onda de luz emitida pelo laser que atinge o anteparo. O índicel do vetor $G$ contém o número de células da matriz $I M_{B I N}$ com valor binário 1 pertencente à colunal. $\mathrm{O}$ valor $G(l)$ representa a amplitude do sinal luminoso na coluna $l$ da matriz $I M_{B I N}$. A Equação (8) foi utilizada para obter o gráfico, sendo $N$ a quantidade de colunas em $I M_{B I N}$.

$$
G(i)=\sum_{\mathrm{j}=1}^{N} \operatorname{IM}_{\mathrm{BIN}}(\mathrm{i}, \mathrm{j})
$$


Distância entre os mínimos locais: o processo para encontrar os mínimos locais considera a simetria do gráfico e a busca foi realizada na metade direita. O ponto máximo de $G$ é facilmente obtido usando a função max (array) do Matlab. Essa função recebe um array de valores e retorna o maior desses valores. A busca pelo primeiro mínimo local à direita inicia do ponto máximo de $G$, denominado $G(k)$, em que $k$ denota o índice de $G$ com maior valor.

Para encontrar o primeiro mínimo local, assume-se $i=k, \mathrm{k} \leq \mathrm{i} \leq \mathrm{M}$ e compara-se o valor de $G(i) \operatorname{com} G(i+1)$. O valor $M$ representa a dimensão de $G$. Inicialmente, espera-se que o valor de $G(i+1)$ seja menor que o valor $\operatorname{de} G(i)$. O valor $G(i+1)$ maior que $G(i)$ indica que a curva do gráfico está, possivelmente, incrementando; logo, o primeiro mínimo local foi encontrado na posição. Ao adotar a referência na posição de máximo, a distância até o primeiro mínimo é, então, dada pela Equação (9).

$$
D=(i-k)
$$

Note-se, ainda, que falsos mínimos locais podem ser encontrados ao invés do mínimo procurado. Isso ocorre devido à descontinuidade do gráfico de intensidade da luz. Para solucionar o problema, apenas os mínimos locais mais significativos são considerados. Dessa forma, passase a assumir que um mínimo local foi encontrado se o valor de $G(i+1)$ é maior que $G(i)$ por um número $\tau$ de vezes, ou seja, se o gráfico se comporta de maneira crescente por um número $\tau$ de vezes. Quando o primeiro incremento ocorrer $G(i+1)<G(i)$, inicializa-se uma variável $c$ com o valor 1 e armazena-se o valor de $i$ em uma variável $i_{\text {min }}$, à medida que novos incrementos consecutivos ocorrem, $c$ vai sendo incrementada. Caso $c$ atinja $\tau$, o mínimo local foi encontrado. Se ao invés de incrementar, $G(i+1)<G(i)$, decrementar, $c$ e $i_{\min }$ passam a receber o valor 0 (zero). Esse fato modifica a Equação (3), e a distância da onda passa a ser calculada pela Equação (10). Sendo que o valor de $D$ é dado em pixels.

$$
D=\left(i_{\min }-k\right)
$$

\section{RESULTADOS E DISCUSSÃO}

Esse estudo utilizou 12 imagens do padrão de difração obtido experimentalmente e duas imagens para cada espessura da fenda. Em seguida, foi calculada a média dos valores da distância entre os mínimos. Cada imagem passou pelos procedimentos descritos anteriormente. A Figura 5 apresenta um exemplo de imagem e respectivo gráfico de intensidade.

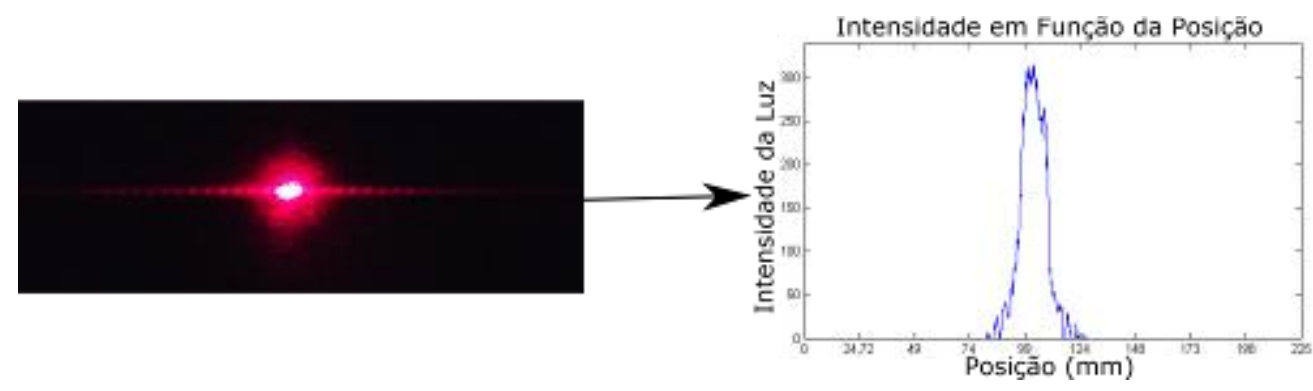

Figura 5: Gráfico da intensidade luminosa a partir de uma imagem. (Fonte: do autor 2015)

A Tabela 1 apresenta a distância entre os mínimos locais obtidos com o algoritmo apresentado, bem como os valores obtidos manualmente e a diferença entre o cálculo manual e o algoritmo a fim de averiguar a desempenho obtido.

Utilizando a Equação (4), pode-se determinar o comprimento de onda $(\lambda)$ do laser, fazendo o ajuste linear dos dados de $\operatorname{Sen}(\theta)$ em função da abertura da fenda (Figura 6). Os valores utilizados no experimento e os valores obtidos manualmente e com o auxílio do algoritmo para o $\operatorname{sen}(\theta)$ são exibidos na Tabela 2. 
Tabela 1: Distância entre os primeiros mínimos locais em função da espessura da fenda

\begin{tabular}{cccc}
\hline Fenda $(\mathrm{mm})$ & $\begin{array}{c}\text { Distância } \\
\text { manual }(\mathrm{mm})\end{array}$ & $\begin{array}{c}\text { Distância } \\
\text { Algoritmo }(\mathrm{mm})\end{array}$ & $\begin{array}{c}\text { Diferença } \\
\left(\mathrm{D}_{\mathrm{m}}-\mathrm{D}_{\mathrm{A}}\right)\end{array}$ \\
\hline 0,1 & 38,0 & 32,28 & 5.72 \\
\hline 0,2 & 25,0 & 21,41 & 3,59 \\
\hline 0,3 & 17,5 & 17,08 & $-0,30$ \\
\hline 0,4 & 15,0 & 16,21 & $-1,21$ \\
\hline 0,5 & 12,0 & 14,48 & $-2,48$ \\
\hline 0,6 & 10.5 & 13,00 & $-2,50$ \\
\hline
\end{tabular}

$\mathrm{O}$ valor do coeficiente angular das retas ajustadas aos dados experimentais representa o comprimento de onda do laser utilizado no experimento. Foram obtidos $1134 \pm 40 \mathrm{~nm}$ e $657 \pm 40$ $\mathrm{nm}$, respectivamente, para os dados obtidos manualmente e com o algoritmo.

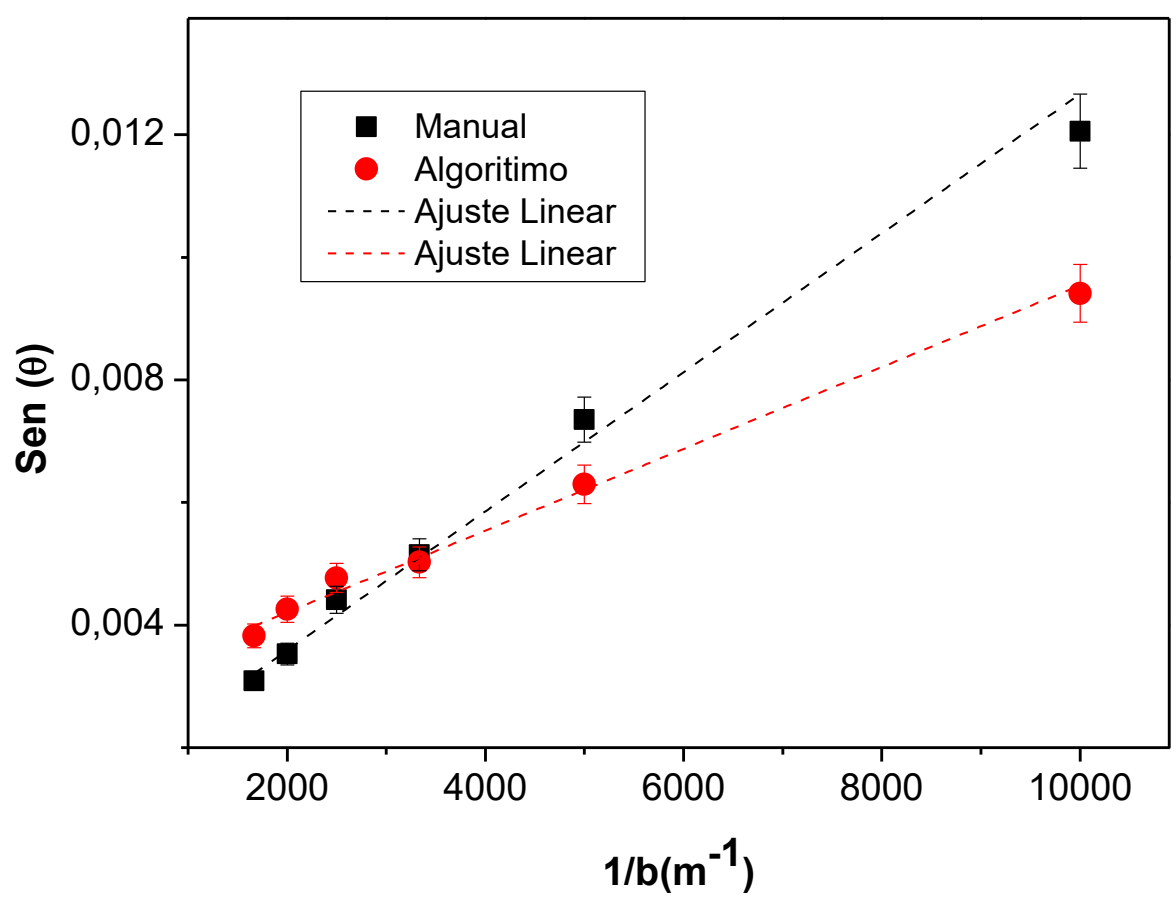

Figura 6: Ajuste linear dos dados experimentais do $\operatorname{Sen}(\theta)$ proporcional a $\Delta p_{y}$ em função de $b^{-1}$. (Fonte: do autor 2015)

O valor nominal do comprimento de onda do laser fornecido pelo fabricante era de $670 \pm 10$ $\mathrm{nm}$. Claramente, os dados obtidos utilizando o Matlab se revelaram em melhor conformidade com o valor esperado.

Tabela 2: Valores experimentais obtidos a partir da imagem do padrão de difração

\begin{tabular}{llll}
\hline $\operatorname{Sen}(\theta)_{\operatorname{man}}$ & $\operatorname{Sen}(\theta)_{\mathrm{Alg}}$ & $1 / \mathrm{b}(\mathrm{m}-1)$ & $(\mathrm{mm})$ \\
\hline 0,01127 & 0,00949 & 10000,000 & $(0,10 \pm 0,03)$ \\
\hline 0,00744 & 0,00638 & 5000,000 & $(0,20 \pm 0,03)$ \\
\hline 0,00522 & 0,00509 & 3333,333 & $(0,30 \pm 0,03)$ \\
\hline 0,00447 & 0,00483 & 2500,000 & $(0,40 \pm 0,03)$ \\
\hline 0,00358 & 0,00432 & 2,000 & $(0,50 \pm 0,03)$ \\
\hline 0,00063 & 0,00388 & 1,666 & $(0,60 \pm 0,03)$ \\
\hline
\end{tabular}


A Figura 7 apresenta o gráfico da intensidade luminosa do padrão de difração obtida no anteparo com o auxílio do Matlab. É possível testar a plausibilidade da Equação 6, mostrando que os dados experimentais estão de acordo com o princípio da incerteza, para isso, tomou-se a distância entre o máximo da curva e os três primeiros valores de mínimos.

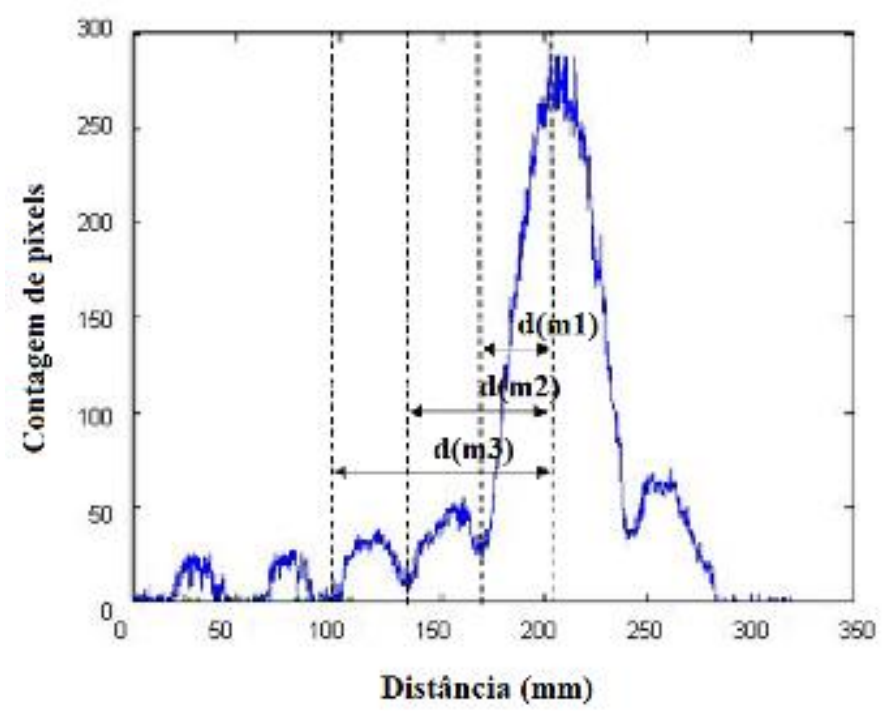

Figura 7: Intensidade luminosa no anteparo para a difração por fenda simples. (Fonte: do autor 2056)

Sabendo que $\Delta p_{y i}=h \operatorname{Sen}(\theta i) / \lambda$, pela Equação 4, tem-se que, $\operatorname{com}(\mathrm{i}=1,2$ e 3$)$. Foram tomados os três primeiros mínimos $\mathrm{i}=1,2$ e 3 . A Tabela 3 exibe os valores teóricos e experimentais obtidos para $\mathrm{m}_{\mathrm{i}}$.

Tabela 3: Valores experimentais obtidos a partir da imagem do padrão de difração

\begin{tabular}{ccc}
\hline $\mathrm{m}_{\mathrm{i} \text { (teórico) }}$ & $\mathrm{m}_{\mathrm{i} \text { (exp.) }}$ & $\operatorname{Sen}\left(\theta_{i}\right)$ \\
\hline 1 & 1.4 & 0,0094 \\
\hline 2 & 2.5 & 0,0169 \\
\hline 3 & 3,6 & 0,0240
\end{tabular}

Os valores experimentais obtidos para os três primeiros mínimos divergem em média por $25 \%$ dos valores esperados. Considerando que a espessura da fenda tem uma incerteza instrumental da ordem de $10 \%$ do valor da abertura utilizado, considerando ainda a dificuldade em manter o paquímetro perpendicular ao feixe do laser e também que a incerteza associada à determinação experimental do comprimento de onda do laser é da ordem de $10 \%$, é possível admitir que esse resultado esteja em razoável concordância com a expectativa teórica. Seria possível tornar mais preciso os valores experimentais de $\mathrm{m}_{\mathrm{i}}$ utilizando um laser cujo comprimento de onda fosse determinado com maior precisão, e uma rede de difração ao invés de um paquímetro, entretanto em favor da utilização do paquímetro pesam dois fortes argumentos, o baixo custo e a possibilidade dada ao estudante de controlar a variável de espessura da fenda e estudar o comportamento do padrão de difração em função da abertura do paquímetro.

\section{CONCLUSÃO}

O experimento proposto é considerado de baixo custo, devido à fácil aquisição dos materiais envolvidos e permitiu o estudo de temas de física moderna, como difração e princípio da incerteza. Também foram utilizados recursos tecnológicos relacionados à programação e processamento de imagens, possibilitando a oportunidade de trabalhar com diversos conceitos da Física Quântica durante o desenvolvimento da atividade. Foi determinado o valor do comprimento de onda do laser utilizado no experimento, o valor experimental obtido via algoritmo de tratamento da 
imagem, para o comprimento de onda foi de $657 \pm 40 \mathrm{~nm}$, e mostrou-se mais coerente com o valor nominal que a mesma grandeza calculada com o auxílio da régua. Com base no cálculo experimental do comprimento de onda, foi verificado o princípio da incerteza, assim, determinando os valores experimentais de $m_{i}$, a diferença entre os valores experimentais obtidos e o valor esperado se revelou dentro das incertezas associadas às medidas experimentais e precisão dos equipamentos.

Os autores consideram que o entendimento da física moderna aparece como uma necessidade para compreender os fatos, os equipamentos e a tecnologia do cotidiano dos estudantes. Mesmo diante da fragilidade dos conhecimentos de física clássica apresentados por muitos alunos, não devemos aceitar a ideia que tende a julgar os jovens como incapazes de perceber a lógica quântica antes de dominarem todo instrumental clássico. Sendo assim, com o intuito de colaborar com o debate acerca da inserção de temas atuais da física no ensino de ciências, o presente trabalho propõe uma atividade experimental, que embora ainda não tenha sido implementada em sala de aula, é relevante para auxiliar o planejamento de atividades voltadas para o ensino de tópicos de física moderna.

Como sugestão de possíveis propostas de aplicação do experimento relatado neste artigo, destacamos a realização de uma atividade de caráter investigativo ofertada para alunos do ensino médio integrado do curso técnico em informática estimulando a turma a comparar os resultados obtidos a partir do algoritmo e da medida direta feita com a régua, ou uma possível aplicação conceitual em turmas da educação básica, nesse caso o algoritmo disponibiliza graficamente a intensidade do padrão de difração em função da distância, o que permite uma boa visualização dos dados obtidos.

\section{REFERÊNCIAS BIBLIOGRÁFICAS}

1. Nussenzveig HM. Curso de Física básica, 4th ed. Vol 1. Ótica Relatividade Físca Quantica. São Paulo: Edgard Blucher; 2002. $437 \mathrm{p}$.

2. Martins TMG, Ribeiro AN, Oliveira DC, Ferreira NS, Santos RA, Santana VM, Macêdo MA. Visualização do Processo Carga Descarga em Capacitores. Scientia Plena [Internet]. 2005 [cited 2016 mar 24]; 1(1): 38-41. Avaliable from: http://www.scientiaplena.org.br/sp/article/view/564.

3. Eisberg RM, Resnick R. Física Quântica: Átomos, Moléculas, Sólidos e Partículas. Rio de Janeiro: Campus; $1979.928 \mathrm{p}$.

4. Feldens B, Dias PMC, Santos WMS. E Assim se Fez o Quantum. Revista Brasileira de Ensino de Física [Intenet]. 2010 [cited 2016 mar 24]; 32(2): 1-11. Avaliable from: http://www.scielo.br/pdf/rbef/v32n2/v32n2a15.pdf. 10.1590/S1806-11172010000200015.

5. Klassen S. The Photoelectric Effect: Reconstructing the Story for the Physics Classroom. Science \& Education. 2011; 20(78): 719-731.

6. Eisberg RM. Fundamentals of Modern Physics. Nova York: John Wiley \& Sons; 1961.729 p.

7. Abrego JRB, Yamaguchi DA, Liboni TA, Barbosa AA, Salinas MBPR, BOSSA GV. Montagem de um conjunto experimental destinado à verificação do princípio da incerteza de Heisenberg. Revista Brasileira de Ensino de Física [Internet]. 2013 [cited 2016 mar 24]; 35(3): 1-5. 10.1590/S180611172013000300012.

8. Cavalcante MA, Jardim V, Barros JAA. Inserção de Física Moderna no Ensino Médio: Difração de um feixe de Laser. Caderno Catarinense de Ensino de Física [Internet]. 1999 [cited 2016 mar 24];16(2): 154-169. 10.5007/6805.

9. Matlab Software version 8.3.0.532 (R2014a) [computer program]. Natick, Massachusetts: The MathWorks Inc; 2014.

10. Otsu NA. Threshold Selection Method from Gray-Level Histograms. IEEE Transactionson Systems, Man, and Cybernetics. 1979; 9(1): 62-66. 10.1109/TSMC.1979.4310076.

11. Chapman SJ. MATLAB Programming for Engineers. Boston: Cengage Learning; 2004.

12. Griffiths DJ. Introduction to Quantum Mechanics, 2nd ed. [place unknown]:Prentice-Hall; 2005.

13. Reis DM, Santos EM, Andrade-Neto AV. Padrão de difração de um conjunto de $\mathrm{n}$ fendas não simétricas e de larguras arbitrárias. Revista Brasileira de Ensino de Física [Internet]. 2015 [cited 2016 jun 20]; 37(2): 2312-1-2312-9. 10.1590/S1806-11173721803.

14. Souza, AMC. Tópicos de Física Contemporânea. 1st ed.Vol 1. Aracaju: Luz; 2002. 140p.

15. Valadares EC, Moreira AM. Ensinando Física Moderna para o segundo grau: efeito fotoelétrico, laser e emissão de corpo negro. Caderno Catarinense de Ensino de Física, 1998; 15(2): 121-135. 
16. Silva JJO. Algoritmo em Matlab [Internet]. Salgueiro: Instituto Federal do Sertão Pernambucano. 2016 - [cited 2016 jun 20]. Available from: https://github.com/junior-oliveira/laserImg2graphic. 